\title{
ON FINITELY MEAN VALENT FUNCTIONS. II
}

\author{
BY \\ D. C. SPENCER
}

1. We suppose $f(z)$ is regular in $|z|<1$ and denote by $W$ the Riemann domain which is the transform of $|z|<1$ by $f$. We shall say that $f(z)$ has valency $p$ if $f(z)$ takes no value $w$ more than $p$ times. More generally, let $W(R)$ be the area (regions covered multiply being counted multiply) of that portion of $W$ which lies in the circle $|w| \leqq R$; then, if

$$
W(R) \leqq p \pi R^{2}
$$

for all $R>0$, where $p$ is a positive number (not necessarily integral), we shall say that $f(z)$ is $p$ mean valent (p.m.v.) $\left.{ }^{1}\right)$. This paper is a sequel to one of the same title to appear shortly in the Proceedings of the London Mathematical Society $\left({ }^{2}\right)$ in which I have shown that many of the known theorems concerning $p$-valent functions may be extended to the wider class of p.m.v. functions. I discuss here the behavior of p.m.v. functions on paths tending to points on the circumference $|z|=1$.

The theorems which I discuss here remain true under hypotheses somewhat less restrictive than the one stated above. For example, the hypothesis that $W(R) \leqq p \pi R^{2}$ only for $R \geqq R_{0}>0$ would suffice (constants now depending on $R_{0}$ as well as $p$ ). Furthermore, slightly less precise versions of the theorems (with $p$ replaced by $p+\epsilon$ ) could be stated subject to the still weaker condition that

$$
\limsup _{R \rightarrow \infty} \frac{W(R)}{\pi R^{2}} \leqq p
$$

Certain theorems $\left({ }^{3}\right)$ proved elsewhere, however, require the full strength of (1.1) for all $R>0$, and for this reason I have not introduced a new definition here.

2. We begin by expressing the inequality (1.1) in a form more convenient for our purpose. Let $n(r, w)$ be the number of times (necessarily bounded by a constant depending on $r$ ) that $f(z)$ takes on the value $w$ in $|z|<r$; and let us take

Presented to the Society, April 27, 1940; received by the editors October 13, 1939, and, in expanded form, April 4, 1940.

(1) This definition was suggested to me by Professor J. E. Littlewood, to whom I am also indebted for advice in the preparation of the paper.

(2) This paper will be referred to as $V_{1}$.

(3) For example, Theorem 1 of $\mathrm{V}_{1}$. The complication of an additional parameter $R_{0}$ is avoided thereby as well. 


$$
\begin{aligned}
p(r, R) & =\frac{1}{2 \pi} \int_{-\pi}^{\pi} n\left(r, R e^{i \Psi}\right) d \Psi, \\
p(R) & =p(1, R)=\lim _{r \rightarrow 1} p(r, R) .
\end{aligned}
$$

Since $p(r, R)$ is an increasing function of $r, p(R)$ exists (but may be infinite). We have

$$
\begin{aligned}
W(R) & =\lim _{r \rightarrow 1} \int_{0}^{R} \int_{-\pi}^{\pi} n\left(r, \mathrm{R} e^{i \Psi}\right) \mathrm{R} d \mathrm{R} d \Psi \\
& =\int_{0}^{R}\left(\lim _{r \rightarrow 1} \frac{1}{2 \pi} \int_{-\pi}^{\pi} n\left(r, \mathrm{R} e^{i \Psi}\right) d \Psi\right) d\left(\pi \mathrm{R}^{2}\right) \\
& =\int_{0}^{R} p(\mathrm{R}) d\left(\pi \mathrm{R}^{2}\right) .
\end{aligned}
$$

Hence the hypothesis (1.1) may be expressed in the form

$$
\int_{0}^{R} p(\mathrm{R}) d\left(\pi \mathrm{R}^{2}\right) \leqq p \pi R^{2} \quad(R>0) .
$$

3. We shall make frequent use of the following lemma:

LEMma 1. Suppose $s_{1} \geqq s_{2}$. Then the hypothesis

$$
\int_{0}^{R_{1}} p(R) d\left(R^{s_{1}}\right) \leqq p R_{1}^{s_{1}} \quad\left(R_{1}>0\right)
$$

implies

$$
\int_{0}^{R_{1}} p(R) d\left(R^{s_{2}}\right) \leqq p R_{1}^{\delta_{2}} \quad\left(R_{1}>0\right)
$$

but not conversely.

Making some trivial transformations of variable, we see it is enough to show that, if $s \geqq 1$,

$$
\int_{0}^{R_{1}} p_{1}(R) d\left(R^{8}\right) \leqq p R_{1}^{8} \quad\left(R_{1}>0\right)
$$

implies

$$
\int_{0}^{R_{1}} p_{1}(R) d R \leqq p R_{1} \quad\left(R_{1}>0\right),
$$

where $p_{1}(R)=p\left(R^{1 / 82}\right)$, but that (3.4) does not imply (3.3). 
Integrating by parts, we have (dropping subscripts)

$$
\begin{aligned}
\int_{0}^{R_{1}} p(R) d R= & \int_{0}^{R_{1}} p(R) \cdot R^{s-1} \cdot R^{1-s} d R \\
= & {\left[R^{1-s} \int_{0}^{R} p(\mathrm{R}) \cdot \mathrm{R}^{s-1} d \mathrm{R}\right]_{0}^{R_{1}} } \\
& +(s-1) \int_{0}^{R_{1}}\left(\int_{0}^{R} p(\mathrm{R}) \mathrm{R}^{s-1} d \mathrm{R}\right) \cdot R^{-s} d R \\
\leqq & \frac{1}{s} p R_{1}+\frac{(s-1)}{s} p R_{1} \\
= & p\left(R_{1}\right)
\end{aligned}
$$

by (3.3).

On the other hand, the converse implication is false. In fact, take

$$
p(R)= \begin{cases}1, & 2 \mu-1 \leqq R<2 \mu, \mu=1,2, \cdots, \\ 0, & \text { otherwise }\end{cases}
$$

and write $R_{1}=n+\theta$, where $n$ is an integer and $0 \leqq \theta<1$. Then

$$
\begin{aligned}
& \int_{0}^{R_{1}} p(R) d R=\sum_{\mu=1}^{[n / 2]}\{(2 \mu-1)-2 \mu\}+ \begin{cases}0, & n \text { even }, \\
\theta, & n \text { odd }\end{cases} \\
& = \begin{cases}\frac{1}{2} n, & n \text { even, } \\
\frac{1}{2}(n-1)+\theta, & n \text { odd, }\end{cases} \\
& \leqq \frac{1}{2} R_{1} \text {. }
\end{aligned}
$$

Hence (3.4) is satisfied with $p=\frac{1}{2}$. But

$$
\begin{aligned}
\int_{0}^{2 \nu} p(R) d\left(R^{s}\right) & =\int_{0}^{(2 \nu)^{s}} p\left(R^{1 / s}\right) d R=\sum_{\mu=1}^{\nu}\left\{(2 \mu)^{s}-(2 \mu-1)^{s}\right\} \\
& =\frac{1}{2}(2 \nu)^{s}+\frac{1}{4} s(2 \nu)^{s-1}+O\left(\nu^{s-2}\right)>\frac{1}{2}(2 \nu)^{s},
\end{aligned}
$$

if $s>1$ and $\nu>\nu_{0}(s)$. Thus, if $s>1$, (3.3) is false for $R_{1}=2 \nu, \nu>\nu_{0}$. We have shown that the converse of the lemma is false for some function $p(R)$, but not for a $p(R)$ corresponding to an actual Riemann domain. However, the $p(R)$ of the schlicht function which maps the unit circle on the domain shown in Fig. 1 differs as little as we please from the choice (3.5), and for it, therefore, the converse of the lemma is false.

4. Lemma 1 shows that the hypothesis

$$
\int_{0}^{R_{1}} p(R) d\left(R^{8}\right) \leqq p R_{1}^{8} \quad\left(R_{1}>0\right)
$$


is the stronger the larger $s$ is. For the sake of completeness I include the following two theorems (but they may be omitted by the reader if he so desires; they have no bearing on the rest of the theory).

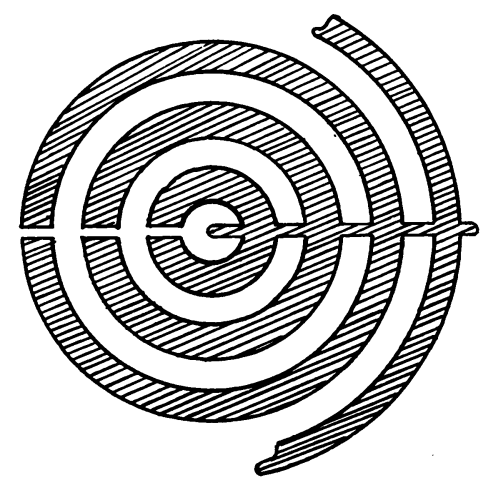

FIG. 1

THEOREM 1. If (s) is true for all $s>0$, and $p(R)$ corresponds to a Riemann domain $W\left({ }^{4}\right)$, then $p(R) \leqq p$.

THEOREM 2. If

$$
f(z)=a_{1} z+a_{2} z^{2}+\cdots
$$

is mean p-valent, so is the (generally algebraic $\left({ }^{5}\right)$ ) function $\left\{f\left(z^{k}\right)\right\}^{1 / k}$. On the other hand, if $k>1$, the mean p-valency of the function

$$
f_{k}(z)=a_{1} z+a_{k+1} z^{k+1}+a_{2 k+1} z^{2 k+1}+\cdots
$$

does not imply that of the function (of form $f_{1}$ )

$$
\left\{f_{k}\left(z^{1 / k}\right)\right\}^{k}=a_{1}^{k} z+k a_{1}^{k-1} a_{k+1} z^{2}+\cdots .
$$

If $f_{k}(z)$ is $p$-valent, then so is $\left\{f_{k}\left(z^{1 / k}\right)\right\}^{k}$. This result and its converse are well known when the functions are $p$-valent $\left({ }^{6}\right)$.

We take Theorem 1 first, and note that if for a given value of $R, R_{0}$ say,

$$
p\left(R_{0}\right)=p\left(1, R_{0}\right)>p,
$$

then, since $p\left(r, R_{0}\right) \rightarrow p\left(R_{0}\right)$ as $r \rightarrow 1$, there exists a $\delta>0$ and $r_{0}=r_{0}(\delta)<1$ such that, for $r>r_{0}$,

(4) The theorem is false if this clause is omitted (and is therefore not trivial).

(5) $\left\{f\left(z^{k}\right)\right\}^{1 / k}$ has branch points at the zeros of $f$ other than the origin. In the neighborhood of the origin, however,

$$
\left\{f\left(z^{k}\right)\right\}^{1 / k}=a_{1}^{1 / k} z+(1 / k) a_{1}^{1 / k-1} a_{2} z^{k+1}+\cdots .
$$

If $f$ is mean 1-valent, then $f$ has at most one zero (by the definition of mean 1-valency), and in this case, therefore, $\left\{f\left(z^{k}\right)\right\}^{1 / k}$ is regular in $|z|<1$ (and so of the form $f_{k}$ ).

(8) See $V_{1}$. 


$$
p\left(r, R_{0}\right)>p+\delta .
$$

We show that this cannot happen.

Suppose it does. Then in the first place $p(r, R)$ is discontinuous at $R=R_{0}$, $q u a$ function of $R$, for each fixed $r>r_{0}$. For if it were continuous we should have $\left({ }^{7}\right)$

$$
\lim _{s \rightarrow \infty} \frac{1}{R_{0}^{s}} \int_{0}^{R_{0}} p(r, R) d\left(R^{s}\right)=\lim _{s \rightarrow \infty} \int_{0}^{1} p\left(r, x R_{0}\right) d\left(x^{s}\right)=p\left(r, R_{0}\right),
$$

which is incompatible with the combination (4.1) and (s) for $R=R_{0}$.

Now let $B(r)$ be the transform of the circumference $|z|=r$ by $f(z)(B(r)$ is the boundary of $W(r)$ ). Then $B(r)$ is an analytic curve, and crosses the circumference $|w|=R_{0}$ a finite (even) number of times if it crosses it at all. If $B(r)$ does not meet $|w|=R_{0}$, or meets it only in points, then it is obvious that $p(r, R)$ is continuous at $R_{0}$. Hence the intersection of $B(r)$ with $|w|=R_{0}$ contains one or more intervals if $r>r_{0}$. These intervals depend upon $r$, but by (4.1) the intervals corresponding to any $r>r_{0}$ have positive total length. It follows that if $r>r_{0}$ the plane measure of $B(r)$ is positive, and so the length (or linear measure) of $B(r)$ is infinite. This is a contradiction of the regularity of $f(z)$ in $|z|<1$, and proves Theorem 1 .

Next, to prove the first part of Theorem 2 , let $p(R), p_{k}(R)$ correspond respectively to $f(z),\left\{f\left(z^{k}\right)\right\}^{1 / k}$. Then

$$
p_{k}(R)=p\left(R^{k}\right) .
$$

In fact, $\{f(z)\}^{1 / k}$ (or branch thereof) maps $|z|<1$ cut along a radius from 0 to 1 on a surface $S$ with function $(1 / k) p\left(R^{k}\right)$; hence $\left\{f\left(z^{k}\right)\right\}^{1 / k}$ (which maps $|z|<1$ on $S$ covered $k$-times) has for function

Finally

$$
k \cdot(1 / k) p\left(R^{k}\right)=p\left(R^{k}\right) .
$$

$$
\int_{0}^{R_{1}} p_{k}(R) d\left(\pi R^{2}\right)=\int_{0}^{R_{1}} p\left(R^{k}\right) d\left(\pi R^{2}\right)=\int_{0}^{R_{1}^{k}} p(R) d\left(\pi R^{2 / k}\right) \leqq p \pi R^{2},
$$

by the mean $p$-valency of $f$ and Lemma 1 . This proves the first half of the theorem.

As for the second half, let $p_{1}(R), p_{k}(R)$ correspond respectively to $f_{1}$ $=\left\{f_{k}\left(z^{1 / k}\right)\right\}^{k}, f_{k}(z)$. Then

$$
p_{1}(R)=p_{k}\left(R^{1 / k}\right) .
$$

An argument similar to that given above to prove the nega tive part of Lemma 1 now shows that there exist mean $p$-valent functions $f_{k}$ and arbitrarily large $R_{1}$ for which

${ }^{(7)}$ Since $x^{8}$ increases practically from 0 to 1 in an arbitrarily small neighborhood of $x=1$ when $s$ is large. 


$$
\int_{0}^{R_{1}} p_{1}(R) d\left(\pi R^{2}\right)=\int_{0}^{R_{1}} p_{k}\left(R^{1 / k}\right) d\left(\pi R^{2}\right)=\int_{0}^{R_{1}^{1 / k}} p_{k}(R) d\left(\pi R^{2 k}\right)>p \pi R_{1}^{2},
$$

if $k>1$. If, however, $p_{k}(R) \leqq p$, then $p_{1}(R) \leqq p$, and in this case (in particular if $f_{k}$ is $p$-valent) $f_{1}$ is mean $p$-valent.

5. After these preliminaries we now study the rate of growth of mean $p$-valent functions. The method depends on the distortion theory of Ahlfors $\left({ }^{8}\right)$, a theory which has already been applied by Cartwright $\left({ }^{9}\right)$ to obtain an upper bound of $M(r, f)$ (the maximum modulus of $f(z)$ on $|z|=r$ ) for $p$-valent functions. By $K(\alpha, \beta, \cdots)$ we denote a positive number depending on the parameters shown explicitly. If it is clear on what parameters $K$ depends, as of ten happens, we simply write $K$. $K$ 's will not necessarily be the same in different contexts.

It is convenient to suppose first that $f(z)$ is regular for $|z| \leqq 1$. We write $w_{0}=f(0)$. Let $C(R)$ be the circumference $|w|=R$ in the $w$-plane, and let $E(R)=W \times C(R)$, the set of points common to $W$ and $C(R)$ (so that $m E(R)$ $=2 \pi R p(R))$. Two points of $E(R)$ are considered distinct if they correspond to distinct sheets of $W$, even though they have the same projection on the complex w-plane. $E(R)$ consists of a finite set of arcs $\left\{I_{\nu}(R)\right\}\left({ }^{10}\right)$, $(\nu=1,2, \cdots, N)$, where $N$ depends on $R$ (and $f$ ). For fixed $R_{1}$ let $r_{\nu}\left(R_{1}\right)$ be the value of $r$ for which $B(r)$ (the transform of $|z|=r$ by $f$ ) just touches $I_{\nu}\left(R_{1}\right)$ (for the first time). If $\left|w_{0}\right|<R<R_{1}$, at least one arc of $E(R)$ separates $I_{\nu}\left(R_{1}\right)$ from $w_{0}$; if more than one, let $I_{\nu}(R)$ be the first which is met in describing a continuous curve lying in $W$ and connecting $w_{0}$ with a point of $I_{\nu}\left(R_{1}\right)$. Let $m I_{\nu}(R)=\Theta_{\nu}(R)$.

Theorem 3. Suppose that $0<r<1$, and that $R_{1}>M\left(r_{0}, f\right)$. Then

$$
2 \pi \int_{M\left(r_{0}, f\right)}^{R_{1}} \frac{d R}{\Theta_{\nu}(R)} \leqq \log \frac{1}{\left(1-r_{\nu}\left(R_{1}\right)\right)^{2}}+K\left(r_{0}\right), \quad\left(\nu=1,2, \cdots, N\left(R_{1}\right)\right) .
$$

Take $R_{1}=M(r, f)$, and let $I_{\nu(r)}$ be any one of the intervals $\left\{I_{\nu}\left(R_{1}\right)\right\}$ which is touched by $B(r)$ (there is at least one). Then, if $r>r_{0}$, we have by Theorem 2 (with $R_{1}=M(r, f), \nu=\nu(r)$ )

$$
2 \pi \int_{M\left(r_{0}, f\right)}^{M(r, f)} \frac{d R}{\Theta_{\nu(r)}(R)} \leqq \log \frac{1}{(1-r)^{2}}+K\left(r_{0}\right) .
$$

This formula has been proved in effect by Cartwright [3]. I omit the proof of the more general formula (5.1) since no essentially new ideas are involved.

\section{Let}

(8) Ahlfors [1].

(9) Cartwright [3].

${ }^{(10)} C(R)$ may not cut $B$ (the transform of $|z|=1$ and the boundary of $W$ ), in which case each interval of $E(R)$ is the whole of $C(R)$, and the number of intervals is the number of sheets cut by $C(R)$ (zero for large $R$ ). 


$$
f_{k}(z)=a_{1} z+a_{k+1} z^{k+1}+\cdots .
$$

We deduce the following theorem from Theorem 3 :

THEOREM 4. If $f_{k}(z)$ is mean p-valent and $M\left(r, f_{k}\right)$ is the maximum modulus of $f_{k}$ on the circle $|z|=r$, then

$$
M\left(r, f_{k}\right) \leqq K(p, k) \mu_{[p / k]}(1-r)^{-2 p / k},
$$

where

$$
\mu_{[p / k]}=\max \left(\left|a_{1}\right|, \cdots,\left|a_{[p / k]}\right|\right) .
$$

Theorem 4 was stated without proof in $V_{1}$. It is known for $p$-valent functions, the case $k=1$ having been proved by Cartwright (loc. cit.); and the general case is an easy deduction from the case $k=1$ when $f_{k}$ is $p$-valent $\left({ }^{11}\right)$. By combining Theorem 4 above with Theorem 3 of $V_{1}$ we obtain the following theorem (also stated without proof in $\mathrm{V}_{1}$ ):

THEOREM 5. If $f_{k}(z)$ is mean p-valent, then

$$
\left|a_{n}\right| \leqq K(p, k) \mu_{[p / k]} n^{2 p / k-1}
$$

provided $p>\frac{1}{4} k$.

Theorem 5 for $p$-valent functions was proved in $\mathrm{V}_{1}\left({ }^{12}\right)$. The restriction that $p>\frac{1}{4} k$ is necessary. In fact, if $n \geqq 1$, take

$$
a_{n k+1}= \begin{cases}\frac{1}{\nu\left(\lambda_{\nu}\right)^{1 / 2}} z^{n k+1}, & \text { if }\left|(n k+1)-\lambda_{\nu}\right| \leqq k / 2 \text { and } \lambda_{\nu} \geqq n k+1, \\ 0, & \text { otherwise, }\end{cases}
$$

where $\left(\lambda_{\nu}\right)$ is a rapidly increasing sequence, and take $a_{1}=1$. We suppose that the $\lambda_{\nu}$ satisfy the inequality

$$
\sum_{\nu=1}^{\infty} \frac{1}{\nu\left(\lambda_{\nu}\right)^{1 / 2}} \leqq 1-(\pi / 6)^{1 / 2}
$$

Then $f_{k}(z)$ is zero only at the origin, and each point of the circle $|w|<(\pi / 6)^{1 / 2}$ is covered by $W_{k}$ (the transform of $|z|<1$ by $f_{k}$ ) once and only once. Since the area of $W_{k}$ is less than or equal to $\pi \sum_{\nu=1}^{\infty} 1 / \nu^{2}=\pi^{3} / 6$, we see that $W(R)$ $\leqq \pi^{2} R^{2}$ for $R>0$, so that $f_{k}$ is mean $\pi$-valent. On the other hand, given any function $\psi(n)$ tending steadily to 0 as $n \rightarrow \infty$, we can choose the $\lambda_{\nu}$ such that $\left|a_{n}\right|>\psi(n) / n^{1 / 2}$ for an infinity of $n$. This Gegenbeispiel in modified form was suggested to me by Professor J. E. Littlewood.

(11) For then the function $f_{1}(z)=\left\{f_{k}\left(z^{1 / k}\right)\right\}^{k}$ is $p$-valent. This line of argument is not possible here (see Theorem 2).

${ }^{(12)}$ The theorem for $p$-valent functions was known subject to certain restrictions on $f_{k}$; in $\mathrm{V}_{1}$ these restrictions were removed. 
7. We now prove Theorem 4. We define $\Theta_{\nu}(\rho, R)$, the function of Theorem 3 , in terms of $f_{k}(\rho, z)$, where $\rho<1$. Then we define

$$
\Theta_{\nu}(R)=\lim _{\rho \rightarrow 1} \Theta_{\nu}(\rho, R) \leqq \lim _{\rho \rightarrow 1} 2 \pi R p(\rho, R)=2 \pi R p(R),
$$

since $f_{k}$ is mean $p$-valent. $\Theta_{\nu}(R)$ is thus an integrable function of $R$ (over any finite interval). Now let $W_{k}$ be the transform of $|z|<1$ by $f_{k}$. Since rotation of $W_{k}$ about the origin through an angle $2 \pi / k$ transforms $W_{k}$ into itself, we see that if $T_{0}$ is any "tube" of $W_{k}$ extending to $\infty$, there are $(k-1)$ other tubes $T_{\nu},(\nu=1,2, \cdots,(k-1))$, each identical to $T_{0}$. If, therefore, $\Theta_{\nu}(R)$ is the width of $T_{\nu}$ measured on $C(R)$, we have

$$
\sum_{\nu=0}^{k} \Theta_{\nu}(R)=k \Theta_{0}(R) \leqq m E(R)=2 \pi p(R),
$$

and so

$$
\Theta_{0}(R) \leqq \frac{2 \pi}{k} R p(R)
$$

Hence

$$
\begin{aligned}
2 \pi \int_{M\left(r_{0}\right)}^{M(r)} \frac{d R}{\Theta_{\nu(r)}(R)} & \geqq k \int_{M\left(r_{i}\right)}^{M(r)} \frac{1}{p(R)} \frac{d R}{R}=k \int_{\log M\left(r_{0}\right)}^{\log M(r)} \frac{d R}{p\left(e^{R}\right)} \\
& \geqq k \frac{\left(\log M(r)-\log M\left(r_{0}\right)\right)^{2}}{\int_{\log M\left(r_{0}\right)}^{\log M\left(e^{R}\right) d R}},
\end{aligned}
$$

since (writing $\psi(R)=1 / p\left(e^{R}\right)$ )

$$
(b-a)^{2}=\left(\int_{a}^{b} \psi^{1 / 2} \psi^{-1 / 2} d R\right)^{2} \leqq \int_{a}^{b} \psi d R \int_{a}^{b} \psi^{-1} d R
$$

by Schwarz's inequality. But

$$
\begin{aligned}
\int_{R_{1}}^{R_{2}} p\left(e^{R}\right) d R & =\int_{e^{R_{1}}}^{e^{R_{2}}} p(R) \frac{d R}{R} \\
& =\left[\frac{1}{R} \int_{e^{R_{1}}}^{R} p(\mathrm{R}) d \mathrm{R}\right]_{e^{R_{1}}}^{e_{2}}+\int_{e^{R_{1}}}^{e^{R_{2}}}\left(\int_{0}^{R} p(\mathrm{R}) d \mathrm{R}\right) \frac{1}{R^{2}} d R \\
& \leqq p+p\left(R_{2}-R_{1}\right)
\end{aligned}
$$

by the hypothesis of mean valency $p$ and Lemma 1 (with $s_{1}=2, s_{2}=1$ ). Substituting from (7.2) (with $R_{1}=\log M\left(r_{0}\right), R_{2}=\log M(r)$ ) in (7.1) and using (5.2), we have

$$
\log M(r) \leqq \frac{p}{k} \log \frac{1}{(1-r)^{2}}+K\left(p, k, r_{0}\right)+\log M\left(r_{0}\right) .
$$


Theorem 4 will follow at once from (7.3) (with $r_{0}=\frac{1}{2}$, say) if

$$
M\left(r_{0}, f\right)<K\left(p, k, r_{0}\right) \mu_{[p / k]} .
$$

To prove (7.4) it is sufficient to show that the family of mean $p$-valent functions $f_{k}$ is quasi-normal of order $[p / k]$ at most, and this follows from the definition of mean valency $p$ and the form of $f_{k}\left({ }^{13}\right)$. This completes the proof of Theorem 4.

8. The full strength of the hypothesis of mean valency $p$ is not used in Theorem 4; all that is used is (7.2), and this in the form

$$
\int_{R_{1}}^{R_{2}} p\left(e^{R}\right) d R \leqq p / s+p\left(R_{2}-R_{1}\right)
$$

where $s>0$, is implied by (s). The hypothesis (l) is, in fact, sufficient for the truth of all theorems proved in this paper. Furthermore, only the properties of $W$ in the neighborhood of $\infty$ are relevant. For example, if $W(R) \leqq p \pi R^{2}$ only for $R>R_{0}>0$, then

$$
M(r, f)=O(1-r)^{-2 p},
$$

where the constant implied in the $O$ depends on $R_{0}, f$, and $p$. More generally if

$$
\limsup _{R \rightarrow \infty} \frac{W(R)}{\pi R^{2}} \leqq p
$$

then, for every $\epsilon>0$,

$$
M(r, f)=O(1-r)^{-2 p-\epsilon} .
$$

Moreover, if (8.1) is satisfied with $p=0$, then

$$
M(r, f)=O(1-r)^{-\epsilon} .
$$

We thus obtain, in particular, the striking result that a schlicht function which fills only an infinitesimal part of the w-plane is of infinitesimal order.

9. We shall say that a set of points in a domain $D$ is a path $P$ if it is a Jordan curve. If the equation of $P$ is

$$
P(t)=x(t)+i y(t),
$$

where $t$ varies from 0 to 1 , and if, given $\epsilon$,

$$
|P(t)-a|<\epsilon
$$

for $t_{0}(\epsilon)<t<1$, then we say $a$ is an end of $P$, or that $P$ converges to the point $a$. A path in $|z|<1$ with end $e^{i \theta}$ will be denoted by $P(\theta)$.

(13) See Montel $[5$, p. 73]. The test given there for quasi-normality $[p / k]$ is satisfied if applied to the functions $f_{k}\left(z^{1 / k}\right)$, which are regular in the unit circle slit along a radius, and this implies that the family $f_{k}(z)$ is quasi-normal of order $[p / k]$. 
THEOREM 6. Suppose that $f(z)$ is p.m.v. and that $E_{\theta}$ is a set of distinct points. If to each point $\theta$ of $E_{\theta}$ there corresponds at least one path $P(\theta)$ for which

$$
\lim _{P_{\theta}} \inf (1-r)^{\alpha(\theta)}|f(z)|>0,
$$

then

$$
\sum_{E_{\theta}} \alpha(\theta) \leqq 2 p
$$

It is sufficient to prove the theorem for an enumerable set $\left(\theta_{v}\right)\left({ }^{14}\right)$. It is then enough to show that

$$
\sum_{\nu=1}^{n} \alpha_{\nu} \leqq 2 p
$$

where $\alpha_{\nu}=\alpha\left(\theta_{\nu}\right)>0$. Under these circumstances there correspond to $R>R_{0}(n, f)$, $n$ arcs $I_{\nu}(R),(1 \leqq \nu \leqq n)$, such that the transform of $I_{\nu}(R)$ by $z=f^{-1}(w)$ is a cross section $\left.{ }^{15}\right) \gamma_{\nu}(R)$ of the unit circle separating the point $e^{i \theta_{\nu}}$ from the origin and converging to $e^{i \theta_{\nu}}$ as $R \rightarrow \infty\left({ }^{16}\right)$. Let $R_{\nu}(r)$ be the largest $R$ for which $\gamma_{\nu}(R)$ has points in common with the circle $|z|=r$, and write

$$
m I_{\nu}(R)=\Theta_{\nu}(R)=2 \pi R \Xi_{\nu}(R) .
$$

Then

$$
2 \pi \int_{K}^{R_{\nu}(r)} \frac{d R}{\Theta_{\nu}(R)}=\int_{K_{1}}^{\log R_{\nu}(r)} \frac{d R}{\Xi_{\nu}\left(e^{R}\right)} \geqq \frac{\left(\log R_{\nu}(r)-R_{1}\right)^{2}}{\int_{R_{1}}^{\log R_{\nu}(r)} \Xi_{\nu}\left(e^{R}\right) d R},
$$

as in the proof of Theorem 4 . That is to say,

$$
\int_{K_{1}}^{\log R_{\nu}(r)} \Xi_{\nu}\left(e^{R}\right) d R \geqq \frac{\left(\log R_{\nu}(r)-R_{1}\right)^{2}}{2 \pi \int_{K}^{R_{\nu}(r)} d R / \Theta_{\nu}(R)} \geqq \frac{\left(\log R_{\nu}(r)-K_{1}\right)^{2}}{\log 1 /(1-r)^{2}+K}
$$

by Theorem 3,

$$
\geqq \frac{1}{2} \alpha_{\nu} \log R_{\nu}(r)+o\left(\log R_{\nu}(r)\right),
$$

by the hypothesis (9.1). This inequality may be written in the form

$$
\frac{1}{2} R \alpha_{\nu} \leqq \int_{K}^{R} \Xi_{\nu}\left(e^{\mathrm{R}}\right) d \mathrm{R}+o(R) .
$$

Summing over $\nu$ from 1 to $n$, we obtain

(14) But even a schlicht function may tend to $\infty$ at a non-enumerable set of discrete points $e^{i \theta}$.

(15) By a cross section of a domain $D$ we mean a path lying in $D$ (except for its end-points) and connecting two distinct boundary points of $D$.

(16) That is, given $\epsilon, \gamma_{\nu}(R)$ lies in a circle of radius $\epsilon$ and center $e^{i \theta_{\nu}}$ if $R>R_{0}(\epsilon)$. The statement is intuitive, and in any case is covered by familiar arguments. 


$$
\frac{1}{2} R \sum_{\nu=1}^{n} \alpha_{\nu} \leqq \int_{K}^{R} \sum_{\nu=1}^{n} \Xi_{\nu}\left(e^{\mathrm{R}}\right) d \mathrm{R}+o(R) \leqq \int_{K}^{R} p\left(e^{\mathrm{R}}\right) d \mathrm{R}+o(R),
$$

since $\sum_{\nu=1}^{n} \Xi_{\nu}(R) \leqq p(R)$,

$$
\leqq p R+o(R)
$$

by the hypothesis of mean valency $p$ (see (7.2)). Dividing by $R$ and letting $R \rightarrow \infty$, we obtain (9.3), and (since $n$ is arbitrary) this proves the theorem.

10. Theorem 7. Suppose $f(z)$ is p.m.v. and that

$$
f(z)=O(1)
$$

on some path $P_{1}\left(\theta_{0}\right)$. Then on any path $P\left(\theta_{0}\right)$

$$
\lim \sup (1-r)^{2 p}|f(z)|=0 .
$$

We suppose there is an infinite sequence of points, $\left(z_{n}\right)$ say, tending to $e^{i \theta_{0}}$ and a number $K>0$ such that $\left|f\left(z_{n}\right)\right|>\left|f\left(z_{n-1}\right)\right|$, and

$$
\left|f\left(z_{n}\right)\right|>K\left(1-r_{n}\right)^{-2 p}, \quad\left|z_{n}\right|=r_{n} ;
$$

we argue by reductio ad absurdum.

Suppose first that there exists an arbitrarily large $R$ such that the transform of $E(R)$ by $z=f^{-1}(w)$ contains an infinity of nonoverlapping cross sections $\gamma_{\nu}(R)$ of $|z|<1$ converging to $e^{i \theta_{0}}$ as $\nu \rightarrow \infty\left({ }^{17}\right)$, and that each $\gamma_{\nu}$ separates at least one point $z_{n_{\nu}}$ from the origin. Changing the numeration (if necessary) we may suppose that $\gamma_{\nu}(R)$ separates $z_{\nu}$ from $z=0$. Let $I_{\nu}(R)$ be the transform by $f(z)$ of $\gamma_{\nu}(R)$, and write $m I_{\nu}(R)=\Theta_{\nu}(R)=2 \pi R \Xi_{\nu}(R), R_{\nu}=\left|f\left(z_{\nu}\right)\right|$. Then

$$
p \log R_{\nu} \leqq \frac{\left(\log R_{\nu}-K\right)^{2}}{2 \pi \int_{K}^{R_{\nu}} d R / \Theta_{\nu}(R)}+O(1)
$$

Otherwise

$$
\log R_{\nu}<2 \pi p \int_{K}^{R_{\nu}} \frac{d R}{\Theta_{\nu}(R)}+O(1)<\log \frac{1}{\left(1-r_{\nu}\right)^{2}}+O(1)
$$

by Theorem 3, and this contradicts the hypothesis (10.3). But (as in the proof of Theorem 6)

$$
\frac{\left(\log R_{\nu}-K\right)^{2}}{2 \pi \int_{K}^{R_{\nu}} d R / \Theta_{\nu}(R)} \leqq \int_{K}^{\log R_{\nu}} \Xi_{\nu}\left(e^{R}\right) d R,
$$

and so, substituting in (10.4),

(17) But no $\gamma_{\nu}$ separates $e^{i \theta_{0}}$ from the origin. 


$$
p \log R_{\nu} \leqq \int_{K}^{\log R_{\nu}} \Xi_{\nu}\left(e^{R}\right) d R+O(1)
$$

Finally, since $\gamma_{\nu-1}$ and $\gamma_{\nu}$ are nonoverlapping, we see that $\Xi_{\nu-1}(R)$ and $\Xi_{\nu}(R)$ are distinct for all (sufficiently) large $R$, and so

$$
\begin{aligned}
\int_{K}^{\log R_{\nu}} \Xi_{\nu}\left(e^{R}\right) d R & \leqq \int_{K}^{\log R_{\nu}} p\left(e^{R}\right) d R-\int_{K}^{\log R_{\nu}} \Xi_{\nu-1}\left(e^{R}\right) d R \\
& \leqq p \log R_{\nu}-p \log R_{\nu-1}+O(1)
\end{aligned}
$$

by the hypothesis of mean valency $p$ and (10.5) for $\nu-1$. (10.5) and (10.6) give a contradiction if $\nu>\nu_{0}$, and so the infinity of nonoverlapping cross sections with the properties stated cannot exist. The alternative is that for $R>R_{0}$ one cross section, $\gamma(R)$ say, separates all but a finite number of $\left(z_{\nu}\right)$ from $z=0$.

Now we can find a number $R_{1}$ such that, for $R>R_{1}, \gamma(R)$ does not separate $e^{i \theta_{0}}$ from $z=0$. Otherwise there would exist no path $P_{1}\left(\theta_{0}\right)$ on which $f=O(1)$, contrary to the hypothesis of the theorem. Since, on the other hand, $\gamma(R)$ separates all but a finite number of the $\left(z_{v}\right)$ from $z=0$, we see that, for $R>R_{1}, \gamma(R)$ has $e^{i \theta_{0}}$ as one end-point. This, I say, is impossible $\left({ }^{18}\right)$. In fact, suppose $R_{1}<R_{2}<R_{3}$, and connect $\gamma\left(R_{2}\right)$ with $\gamma\left(R_{3}\right)$ by a simple analytic curve lying in $|z|<1$. Let $q_{1}$ be the last intersection of this curve with $\gamma\left(R_{2}\right), q_{2}$ the first intersection with $\gamma\left(R_{3}\right)$. Then the portion $P$ of the curve connecting $q_{1}$ with $q_{2}$ lies in a sub-domain $D$ of $|z|<1$ (bounded by $\gamma\left(R_{2}\right), \gamma\left(R_{3}\right)$, and points of $|z|=1)$, and divides $D$ into two domains. Let $D_{1}$ be the domain bounded by $\gamma\left(R_{2}\right), \gamma\left(R_{3}\right), P$, and $e^{i \theta_{0}}$; and let $W_{1}$ be the transform of $D_{1}, \Pi$ the transform of $P$, by $f$. Suppose $R_{2}<R<R_{3}$, and let $I(R)$ be the first cross section of $W_{1}$ on $C(R)$ which is met in describing a continuous curve from $E\left(R_{2}\right)$ to $E\left(R_{3}\right)$ in $W_{1}$. We write $\Theta(R)=m I(R)$. Then, by the hypothesis of mean valency $p$,

$$
\int_{R_{1}}^{R_{2}} m I(R) d R \leqq p \pi R_{2}^{2} \text {. }
$$

Hence, if $K=2 p \pi R_{2}^{2} /\left(R_{2}-R_{1}\right)$, and $E$ is the set of values of $R$ in the interval $R_{1}<R<R_{2}$ for which

then

$$
m I(R)>K
$$

$$
m E<\frac{1}{2}\left(R_{2}-R_{1}\right)
$$

Next, we define

$$
J(R)=\left\{\begin{array}{l}
I(R), \text { if } m I(R) \leqq K, \\
\text { a portion of } I(R) \text { of length } K \text { measured from } \Pi \text { if } m I(R)>K
\end{array}\right.
$$

(18) For finitely mean valent functions, but not for infinitely mean valent functions. 
Let $W_{2}$ be one of the sub-domains of $W_{1}$ swept out by $J(R)$ as $R$ varies from $R_{2}$ to $R_{3}$, which contains, as part of its boundary, a set $A$ of boundary points of $W$ of positive measure. Such a sub-domain exists by (10.7). Further, $W_{2}$ is plainly a finitely valent domain; and every point of its boundary is accessible (by the definition of accessibility). We map $W_{2}$ on a sub-domain $D_{2}$ of $|z|<1$ by $f^{-1}$, the set $A$ corresponding to the boundary point $e^{i \theta_{0}}$. This contradicts well known theorems on the correspondence of boundaries $\left({ }^{19}\right)$ and proves our statement.

11. The conclusion (9.2) of Theorem 6 is a best possible one when $p$ is integral, as shown by the $p$-valent function

$$
f_{n}(z)=\frac{z^{p}}{\left(1+z^{n}\right)^{2 p / n}} .
$$

On the other hand, the hypothesis (9.1) cannot be relaxed to the extent of replacing lim inf by lim sup. We have in fact

THEOREM 8. If $\psi(r)$ is any real function of $r$ satisfying

$$
(1-r)^{2}=o(\psi(r)),
$$

then there is a function $f(z)$ regular and schlicht in $|z|<1$ such that, for at least one path $P\left(\theta_{v}\right)$,

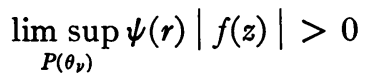

at an enumerable infinity of discrete points $\left(\theta_{v}\right)$.

The following theorem shows that Theorem 7 is best possible.

Theorem 9. Suppose $\psi(r)$ satisfies (11.1). Then there is a schlicht function $f(z)$ such that the radial limit, $\lim _{r \rightarrow 1} f\left(r e^{i \theta}\right)$, exists everywhere and is finite, but

$$
\lim \sup \psi(r)|f(z)|>0
$$

on at least one path $P\left(\theta_{0}\right)$.

The function whose existence is asserted in Theorem 9 is simpler and we discuss it first. We take $f(z)$ to be the function which maps $|z|<1$ on the simply-connected domain $W$ shown in Fig. 2, with $f(0)=0$ and $f^{\prime}(0)$ real and positive (so that $f$ is uniquely defined by $W$ ). $W$ consists of the whole $w$-plane slit along an infinity of concentric circles of radii $R_{\nu},(\nu=1,2, \cdots)$, each annular region $\left(R_{\nu}, R_{\nu+1}\right)$ being connected by a "thin tube" to the interior of the circle of radius $R_{1}$. Every point of the boundary $B$ of $W$ is accessible except points on the line extending from $\omega$ to $\infty$. The line from $\omega$ to $\infty$ is an infinite prime-end with the single accessible nuclear point (Hauptpunkt)

(19) See, for example, Carathéodory [2]. 
$\omega\left({ }^{20}\right)$. Let $e^{i \theta_{0}}$ be the point corresponding to this prime-end by $f^{-1}$. The function $f(z)$ tends to a finite limit on every radius, the limit being, however, unbounded in the neighborhood of $e^{i \theta_{0}}$. We choose (successively) the radii

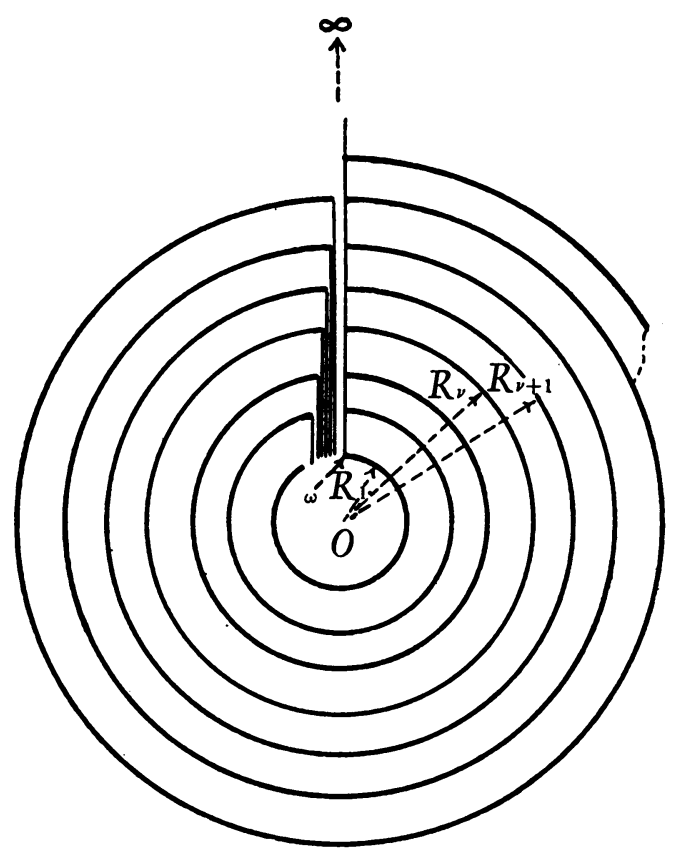

FIG. 2

$\left(R_{v}\right)$ of the figure and construct a path $P\left(\theta_{0}\right)$ whose transform by $f(z)$ approximates to every point of the infinite prime-end and on which (11.3) is satisfied.

We show that the radii $\left(R_{u}\right)$ can be so chosen that

$$
\frac{R_{\nu}+R_{\nu+1}}{2}>\frac{K}{\psi\left(r_{\nu}\right)}
$$

$$
(\nu=1,2, \cdots)
$$

where $r_{\nu}$ is the value of $r$ for which $B(r)$ first touches the circumference $C\left(\frac{1}{2}\left(R_{\nu}+R_{\nu+1}\right)\right)$. Then if $z_{\nu}$ satisfies

$$
f\left(z_{v}\right)=\frac{R_{\nu}+R_{\nu+1}}{2}
$$$$
|z|=r_{\nu}
$$

we have only to connect the $z_{\nu}$ to obtain the desired path $P\left(\theta_{0}\right)$. For if $R>R_{1}$, the set $E(R)$ transforms by $z=f^{-1}(w)$ into a sequence of nonoverlapping cross sections $\left(\gamma_{\nu}(R)\right)$, where $\gamma_{\nu}(R)$ separates $z_{\nu}$ from the origin if $\nu>\gamma_{0}(R)$. Since

${ }^{(20)}$ See Carathéodory [2]. 
$\gamma_{\nu}(R)$ converges to $e^{i \theta_{0}}$ as $\nu \rightarrow \infty, z_{\nu}$ tends to the same point (as $\left.\nu \rightarrow \infty\right)$ and

$$
\psi\left(r_{\nu}\right)\left|f\left(z_{\nu}\right)\right|=\psi\left(r_{\nu}\right) \frac{R_{\nu}+R_{\nu+1}}{2}>K
$$

by (11.4).

If, having chosen $R_{\nu}$, we can choose $R_{\nu+1}$ such that (11.4) is satisfied by the function $f_{\nu}(z)$ which maps (with $f_{\nu}(0)=0, f_{\nu}^{\prime}(0)>0$ ) the circle $|z|<1$ on the sub-domain $W_{\nu}$ of $W$ shown in Fig. 3, it will follow by the subordination principle $\left({ }^{21}\right)$ that (11.4) is a fortiori satisfied by $f$ uniformly in $\nu$.

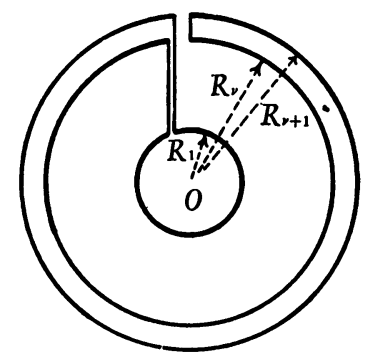

Fig. 3

To show that, for suitable choice of $R_{\nu+1}, f_{\nu}$ satisfies (11.4), we cut $W_{\nu}$ along a radius from 0 to a point $w$ of its boundary on $|w|=R_{1}$, and map the resulting domain by means of $s_{\nu}(z)=\sigma+i \tau=\log w$ on a strip $S_{\nu}$. Now for a parallel strip $U$ defined by

$$
\zeta(z)=\xi+i \eta, \quad \xi_{0}<\xi<\xi_{1},|\eta|<a \pi,
$$

we have

$$
M(r, \zeta) \geqq \log \frac{1}{(1-r)^{2 a}}+K\left(\xi_{0}\right)
$$

if $\xi_{0}+A<M(r, \zeta)<\xi_{1}-A$. But for suitable $z_{0}$ (depending on $R$ ), the function $s_{\nu}(h(z))$, where

$$
h(z)=\frac{z-z_{0}}{z \bar{z}_{0}-1}
$$

is superordinate to a $U$ with $\xi_{0}=\log R_{\nu}, \xi_{1}=\log R_{\nu+1}$ and $a=1-1 / \xi_{1}$ (since we may make the angular spread of the annular region of $W_{\nu}$ as near to $2 \pi$ as we please). Hence

$$
M\left(r, s_{\nu}\right) \geqq M\left(r, s_{\nu}(h)\right)-K\left(R_{\nu}\right) \geqq M(r, \zeta)-K\left(R_{\nu}\right),
$$

by subordination. If $K_{1}\left(R_{\nu}\right)<M(r, \zeta)<\log R_{\nu+1}-K_{1}$, this is not less than

(21) See Littlewood [6]. 


$$
\log \frac{1}{(1-r)^{2}}-\frac{1}{\xi_{1}} \log \frac{1}{1-r}-K\left(R_{\nu}\right),
$$

by (11.5); and is greater than or equal to

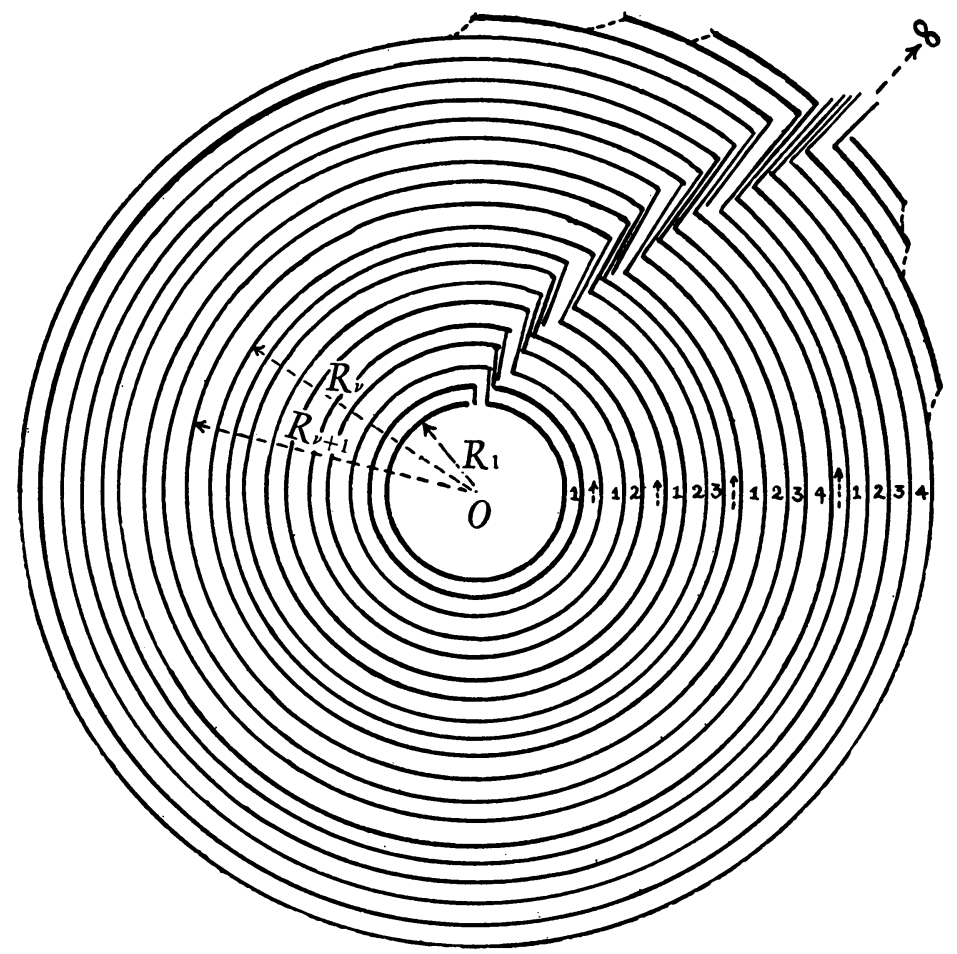

FIG. 4

$$
\log \frac{1}{(1-r)^{2}}-K\left(R_{v}\right) \geqq \log \frac{K}{\psi(r)}
$$

if $r$ (and so $R_{\nu+1}$ ) is large enough. In particular,

$$
\log \frac{R_{\nu}+R_{\nu+1}}{2}=M\left(r_{\nu}, s_{\nu}\right) \geqq \log \frac{K}{\psi\left(r_{\nu}\right)}
$$

if $R_{\nu+1}>R_{0}\left(R_{\nu}\right)$. We can thus choose $R_{\nu+1}$ so that $f_{\nu}$ satisfies (11.4), and this completes the proof of Theorem 7 .

12. In Theorem 8 let $f(z)$ be the function which maps $|z|<1$ on the $w$-plane slit as shown in Fig. 4. The domain consists of an infinity of "tubes" (numbered as shown) connecting the circle $|w| \leqq R_{1}$ with $\infty$. If we write

$$
N(n)=\sum_{\mu=1}^{n} \mu
$$


then the $\nu$ th tube has an angular spread of nearly $2 \pi$ over the "long" intervals (of $R$ ):

$$
R_{N(n)+\nu-1}<R<R_{N(n)+\nu} \quad(n \geqq \nu) .
$$

Using the same notation as in Theorem 9, we see it is enough to show that the radii may be chosen successively in such a way that

$$
\frac{R_{\nu}+R_{\nu+1}}{2}>\frac{K}{\psi\left(r_{\nu}\right)} \quad(\nu=1,2, \cdots) .
$$

The proof is, however, now similar to that given already in the preceding section for the corresponding inequality (11.4), and I omit it.

13. I add finally a theorem of a somewhat different sort:

THEOREм 10. Suppose that $f(z)$ is regular in $|z|<1$, and satisfies the condition that $W(R)<\infty, 0 \leqq R<\infty$. Let $E_{1}(\theta), E_{2}(\theta)$ be the sets of limit points as $f(z)$ tends to $e^{i \theta}$ along two paths $P_{1}(\theta), P_{2}(\theta)$ respectively. Then $E_{1}(\theta) \times E_{2}(\theta)$ $\neq 0\left({ }^{22}\right)$.

This theorem is related to a well known theorem of Lindelöf $\left({ }^{23}\right)$ which states that, if $f$ is bounded in $|z|<1$ and tends to limits $l_{1}, l_{2}$, along two paths $P_{1}(\theta), P_{2}(\theta)$, then $l_{1}=l_{2}$. Theorem 10 is false for bounded functions; there exist (infinitely mean valent) bounded functions such that, for at least one point $e^{i \theta}, E_{1}(\theta) \times E_{2}(\theta)=0\left({ }^{24}\right)$. On the other hand, if $F(\theta)=E_{1}(\theta) \times E_{2}(\theta)$, the hypothesis " $F(\theta) \neq 0$ for all $\theta$ " does not imply the finiteness of $W(R)\left({ }^{25}\right)$, so that the conditions $F(\theta) \neq 0, W(R)<\infty$ are not equivalent.

In proving Theorem 10 we may plainly suppose that $|f|$ is bounded on $P_{1}(\theta), P_{2}(\theta)$ and that $P_{1}, P_{2}$ do not intersect. Then, joining $P_{1}$ to $P_{2}$ by a path $Q$ lying inside $|z|<1$, we can map the sub-domain of $|z|<1$ bounded by $P_{1}, P_{2}$, and $Q$, on to the unit circle, the paths $P_{1}, P_{2}$ being transformed into two arcs, $\Pi_{1}, \Pi_{2}$, abutting at a point $e^{i \theta}$. Let $L_{1}, L_{2}$ be the transforms of $\Pi_{1}, \Pi_{2}$ by $f$, and let $\Lambda_{1}, \Lambda_{2}$ be the projections of $L_{1}, L_{2}$ on the $w$-plane. We suppose $E_{1} \times E_{2}=0$, and argue by reductio ad absurdum.

If $E_{1} \times E_{2}=0$, there exist two positive numbers $\delta$ and $r_{1}$ such that the portions of $\Lambda_{1}$ and $\Lambda_{2}$ corresponding to the arc of $|z|=1$ which lies inside a circle of radius $r_{1}$ and center $e^{i \theta}$ are separated by a distance $\delta$. Let $c(r)$ be that arc of the circle of radius $r$ and center $e^{i \theta}$ which lies in $|z|<1$, and let $\Gamma(r)$ be

(22) That is, $E_{1}$ and $E_{2}$ contain a common point (which may be $\infty$ ).

(23) Lindelöf [4].

(24) An example is the function $f$ which maps the unit circle on the circle $|w| \leqq R$ covered infinitely many times, with winding point at $w=0$. There is then one point $e^{i \theta}$, and two paths $P_{1}, P_{2}$ converging to it, such that the transforms of $P_{1}$ and $P_{2}$ by $f$ are concentric circles.

$\left.{ }^{25}\right)$ In fact, if $f$ maps the unit circle on a Riemann domain bounded by a "spiral" with asymptotic point $w=0$, then $f$ tends to a limit on every path $P(\theta)$. By coiling the spiral sufficiently loosely, the sum of the areas bounded by successive loops can be made infinite. 
that portion of the transform of $c(r)$ which connects $\Lambda_{1}$ to $\Lambda_{2}$. Let $W_{r_{1}}$ be the simply connected domain bounded by $\Gamma\left(r_{1}\right), \cdots, \Gamma\left(r_{1}\right)$, and subsets $B_{1}\left(r_{1}\right)$, $B_{2}\left(r_{1}\right)$ of the boundary continua $\Lambda_{1}$ and $\Lambda_{2}$. Now I say no point $w$ is covered by $W_{r_{1}}$ more than a finite number of times. For, by the construction of $W_{r_{1}}$, the boundary curves $B_{1}\left(r_{1}\right), B_{2}\left(r_{1}\right)$ are both simple, and $\Gamma\left(r_{1}\right)$ (the transform of a portion of $\left.c\left(r_{1}\right)\right)$ is analytic. Hence, if a point $w$ were covered an infinity of times, some neighborhood of $w$ would be covered an infinity of times, and the area of $W_{r_{1}}$ would therefore be infinite, contradicting the hypothesis that $W(R)<\infty$, for finite $R$ (since $W_{r_{1}}$ is a finite domain). Similarly, if $W_{r_{1}}^{\prime}$ is an interior domain, the boundary of which is at distance $\delta / 4$, say, from the boundary of $W_{r}$, then the valency of points of $W_{r}^{\prime}$ is uniformly bounded by a number $K$.

Finally, as $r \rightarrow 0, \Gamma(r)$ converges to an "end" $\xi$ of $W\left(r_{1}\right)$ in the sense of Carathéodory $\left.{ }^{26}\right)$. Since $W\left(r_{1}\right)$ is bounded and $\Lambda\left(r_{1}\right), \Lambda\left(r_{2}\right)$ are separated by a distance $\delta / 2, \Gamma(r)$ cannot converge to a point or to $\infty$. Therefore, $\xi$ is not a prime-end, and so cannot correspond to a single point. This is a contradiction and proves the theorem.

\section{REFERENCES}

1. L. Ahlfors, Untersuchungen zur Theorie der konformen Abbildung und der ganzen Funktionen, Acta Academiae Scientiarum Fennicae, vol. 1 (1930), no. 7.

2. C. Carathéodory, Über die Begrenzung einfach zusammenhängender Gebiete, Mathematische Annalen, vol. 73 (1912), pp. 323-370.

3. M L. Cartwright, Some inequalities in the theory of functions, Mathematische Annalen, vol. 111 (1935), pp. 98-118.

4. E. Lindelöf, Sur un principe général de l'analyse et ses applications à la théorie de la representation conforme, Acta Academiae Scientiarum Fennicae, vol. 46 (1915).

5. P. Montel, Leçons sur les Familles Normales, Paris, 1927.

6. J. E. Littlewood, On inequalities in the theory of functions, Proceedings of the London Mathematical Society, (2), vol. 23 (1925), pp. 481-519.

(26) See Carathéodory [2]. Carathéodory develops his theory only for schlicht functions; here we require its extension to finitely valent functions. The extension is, however, trivial.

Massachusetts Institute of Technology,

Cambridge, Mass. 